\title{
Quantum dot optofluidic lasers and their prospects for biochemical sensing
}

\author{
Alper Kiraz ${ }^{a, b}$, Qiushu Chen ${ }^{b}$, Mehdi Aas ${ }^{a}$, Alexandr Jonášs ${ }^{c}$, and Xudong $\operatorname{Fan}^{b}$ \\ ${ }^{a}$ Department of Physics, Koç University, Rumelifeneri Yolu, Sariyer, 34450 Istanbul, Turkey; \\ ${ }^{b}$ Department of Biomedical Engineering, University of Michigan, Ann Arbor, Michigan 48109, \\ United States; \\ ${ }^{c}$ Department of Physics Engineering, Istanbul Technical University, 34469 Maslak, Istanbul, \\ Turkey
}

\begin{abstract}
We achieved four types of laser emissions with quantum dots (QDs) using the same high-Q-factor optofluidic ring resonator (OFRR) platform. In the first type, $2 \mu \mathrm{M}$ QDs dissolved in toluene that filled the entire OFRR cavity volume were employed as the gain medium. The lasing threshold was $15-22 \mu \mathrm{J} / \mathrm{mm}^{2}$. In the second type, $2 \mu \mathrm{M}$ aqueous QDs were in bulk buffer solution that filled the entire OFRR cavity volume. The lasing threshold was $0.1 \mu \mathrm{J} / \mathrm{mm}^{2}$, over 3 orders of magnitude lower than the state-of-the-art. In the third type, the aqueous QDs were immobilized as a single layer on the interface between the OFRR inner wall and buffer solution with a surface density as low as $3 \times 10^{9}-10^{1} 0 \mathrm{~cm}^{-2}$. The lasing threshold of $60 \mu \mathrm{J} / \mathrm{mm}^{2}$ was achieved. In the fourth type, we achieved optofluidic FRET lasing using aqueous QDs as FRET donors and Cy5 dye molecules as acceptors. We observed lasing from Cy5 emission band in QD-Cy5 pair when excited at QD absorption band, far away from Cy5 absorption maximum. We also report a comprehensive theoretical analysis of optofluidic FRET lasers that was performed based on a Fabry-Perot microcavity using a rate equation model. By comparing FRET lasingbased sensors with conventional sensors using FRET signals obtained by spontaneous fluorescence emission, we show that for optimal pump fluence and FRET pair concentration, FRET lasing can lead to more than 20-fold enhancement in detection sensitivities of conformation changes for linker lengths in the Förster radius range.
\end{abstract}

Keywords: Quantum dot lasers, optofluidic ring resonator, fluorescence resonance energy tranfer, FRET lasers, rate equation model, optofluidic lasers, biosensors, quantum dots

\section{INTRODUCTION}

High sensitivity of stimulated emission to small perturbations in the laser cavity and gain medium enables optofluidic lasers to be exquisite new tools for biosensing applications. ${ }^{1,2}$ Recently, optofluidic laser biosensors have been applied to DNAs, ${ }^{3}$ proteins, ${ }^{4}$ cells, ${ }^{5}$ and tissues ${ }^{6}$ to reveal sub-nanometer conformational changes in biomolecules, ${ }^{7}$ distinguish small thermal dynamic difference between two biomolecules, ${ }^{3}$ analyze structures and morphologies of cells and tissues, ${ }^{6}$ and detect biomarkers at extremely low concentrations $(\sim 1 \mathrm{fg} / \mathrm{mL}),{ }^{8}$ all of which cannot easily be achieved with standard fluorescence techniques based on spontaneous light emission. Analogous to their counterparts used in traditional fluorescence detection, gain medium in both bulk solution ${ }^{3,5,7}$ and on solid/liquid interfaces ${ }^{9}$ have been realized with optofluidic lasers.

Depite the fact that they are suffering from photobleaching, to date, organic dyes have been the most commonly used gain medium for optofluidic lasers. Organic dyes are also sensitive to solvent conditions (such as $\mathrm{pH}$, polarity, and ionic strength), making it less attractive when used in different biological systems. In contrast, semiconductor quantum dots (QDs) come together with unique advantages over organic dyes. Core/shell structured QDs can be engineered with optimized passivation layers to achieve monodisperse sizes while possessing high resistance to photobleaching and high quantum yields in the presence of water and other harsh solvents (high acidity, basicity, and salt concentration, etc. ${ }^{10}$ In addition, QDs have high absorption cross sections, ${ }^{11}$

Further author information: (Send correspondence to A.K.)

A.K.: E-mail: akiraz@ku.edu.tr, Telephone: +90 2123381701

Laser Resonators, Microresonators, and Beam Control XVIII, edited by Alexis V. Kudryashov,

Alan H. Paxton, Vladimir S. Ilchenko, Lutz Aschke, Proc. of SPIE Vol. 9727, 972716

(c) 2016 SPIE CCC code: $0277-786 X / 16 / \$ 18 \cdot$ doi: $10.1117 / 12.2214889$

Proc. of SPIE Vol. $9727972716-1$ 


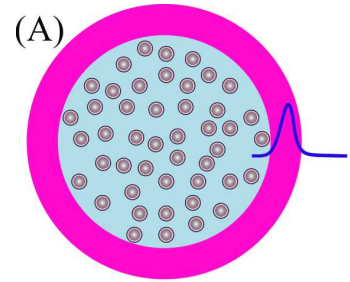

(C)

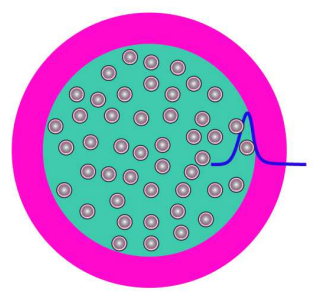

(B)

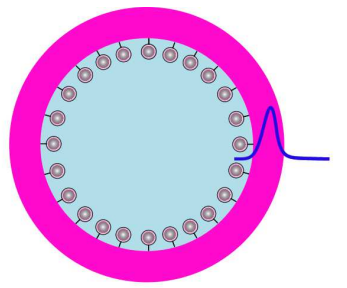

(D)

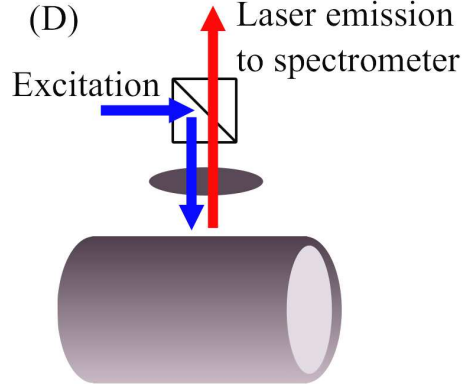

(E)

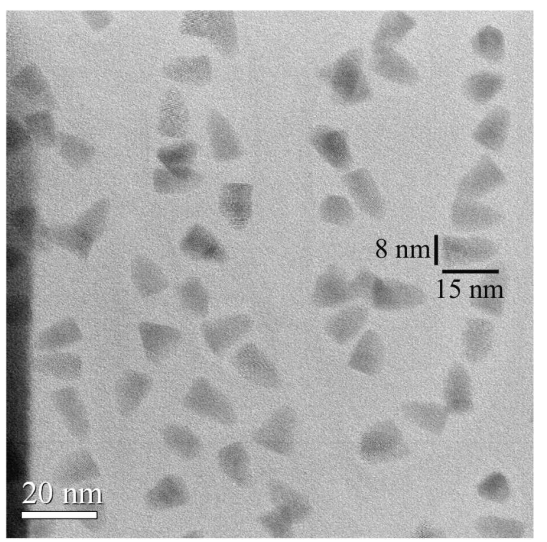

Figure 1. Illustrations of aqueous QDs (A) in solution inside an OFRR, (B) immobilized as a single layer on the inner surface of an OFRR. (C) Illustration of QDs dissolved in toluene solution inside an OFRR. Qualitative sketches of radial dependence of WGM intensity are also shown (A), (B), and (C). (D) Illustration of the experimental setup using confocal geometry. (E) Representative TEM image of Qdot ${ }^{\circledR} 655$ QDs used in this study. Average dimensions are estimated to be $8 \times 15 \mathrm{~nm}$.

which is critical for lasing with low pump intensities. Furthermore, QDs have broad absorption bands and their emission wavelength can be tuned by simply changing their size or composition; different colors of fluorescence can be obtained using the same excitation source. Therefore, QDs have increasingly been used as alternatives to organic dyes for biosensing, imaging, and ion detection applications. ${ }^{12-14}$

Here, we demonstrate low-threshold lasing emission from QDs when they are in bulk aqueous or toluene solutions or immobilized as a single layer on the interface between a solid substrate and aqueous solution. Due to the excellent Q-factor of the optical cavity and the high fluorescence quality of the QDs, lasing thresholds on the order of $0.1 \mu \mathrm{J} / \mathrm{mm}^{2}$ was obtained for QDs in bulk aqueous solution with a concentration as low as $2 \mu \mathrm{M}, 3$ orders of magnitude lower than the state-of-the-art with the similar QD concentration. ${ }^{15}$ For QDs immobilized as a single layer with a surface density as low as $6 \times 10^{9} \mathrm{~cm}^{-2}$, the lasing threshold was approximately 60 $\mu \mathrm{J} / \mathrm{mm}^{2}$. In all cases, the QD lasing persisted even under uninterrupted pulsed pumping 7 times the lasing threshold for 10 minutes, showing significantly higher resistance to photobleaching than organic dyes that were almost completely photobleached within a few minutes of laser operation under similar pumping conditions. We also demonstrate fluoresence resonance energy transfer (FRET) lasing from Cy5 emission band in QD-Cy5 FRET pairs when excited at QD absorption band, far away from Cy5 absorption maximum. ${ }^{16}$ Finally, we use a rate equation model to compare the performance of FRET lasing-based sensors with conventional sensors using FRET signals obtained by spontaneous fluorescence emission. ${ }^{17}$ We show that for optimal pump fluence and FRET pair concentration, FRET lasing can lead to more than 20-fold enhancement in detection sensitivities of conformation changes for linker lengths in the Förster radius range.

\section{EXPERIMENTAL}

The laser cavity used in our work was an optofluidic ring resonator (OFRR) based on a thin-walled fused silica capillary. The fabrication and characteristics of the OFRR have been well studied in the past few years. ${ }^{18}$ Briefly, the OFRR with an inner diameter of 70-90 $\mu \mathrm{m}$ and a wall thickness of 1-2 $\mu \mathrm{m}$ was obtained by rapidly stretching a fused silica pre-form under $\mathrm{CO}_{2}$ laser illumination. The circular cross section of the capillary forms the ring resonator that supports the high $\mathrm{Q}\left(\sim 10^{7}\right)$ whispering gallery modes (WGMs). The OFRR is a very versatile cavity that can accommodate liquids with various refractive indices. ${ }^{19,20}$ When the gain medium is in liquid having a refractive index lower than that of glass, the WGM is mainly confined within the capillary wall, but has an evanescent field present inside the capillary to provide optical feedback for the gain medium 

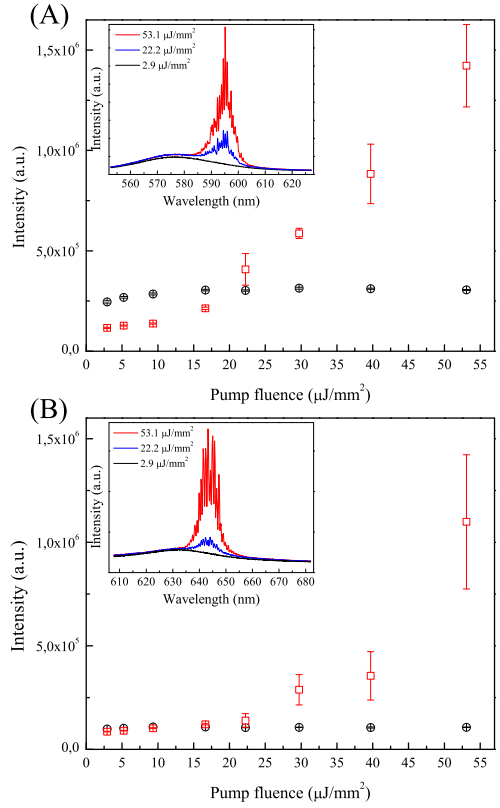
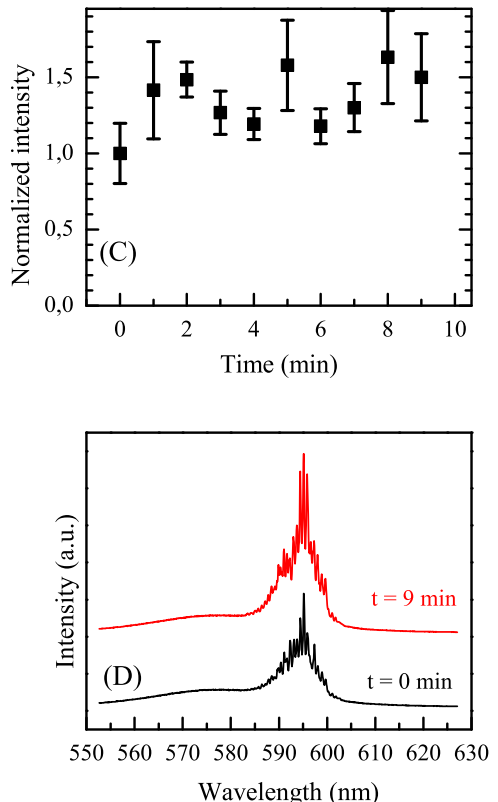
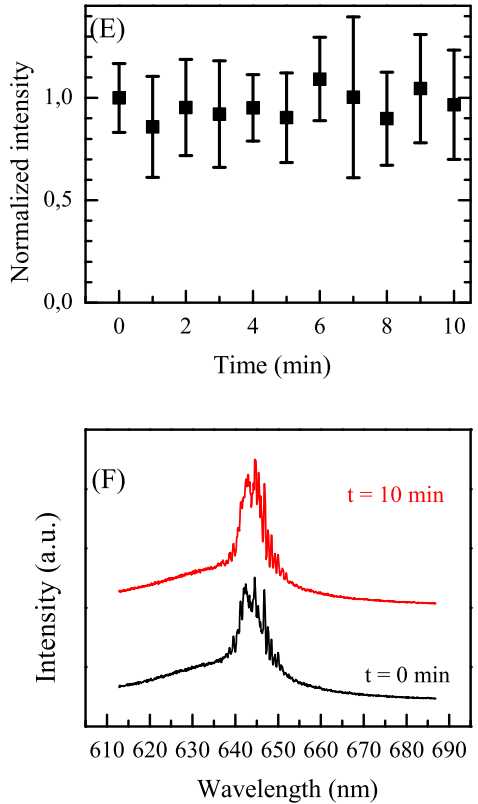

Figure 2. Pump intensity dependence of the spectral intensities recorded from lasing and non-lasing spectral regions in the emission of orange (A) and red (B) emitting QDs dissolved in toluence. Spectral integration takes place in the range of 593-598 $\mathrm{nm}(642-647 \mathrm{~nm})$ for lasing and 575-580 $\mathrm{nm}(620-625 \mathrm{~nm})$ for fluorescence of orange (red) emitting QDs, respectively. Insets show spectra recorded at varying pump fluences. Lasing thresholds are $15 \mu \mathrm{J} / \mathrm{mm}^{2}$ and $22 \mu \mathrm{J} / \mathrm{mm}^{2}$ per pulse for orange and red emitting QDs, respectively. (C) Normalized lasing intensity integrated between 595-598 nm from $2 \mu \mathrm{M}$ orange emitting QDs dissolved in toluene over a duration of 9 minutes under $20 \mathrm{~Hz}$ pumping at an intensity of $3.5 \mathrm{X}$ threshold $\left(55 \mu \mathrm{J} / \mathrm{mm}^{2}\right.$ per pulse). Error bars are obtained with 3 measurements. (D) Lasing spectra recorded from orange emitting QDs dissolved in toluene at $\mathrm{t}=0 \mathrm{~min}$ and $\mathrm{t}=9 \mathrm{~min}$ show negligible photo-bleaching. (E) Normalized lasing intensity integrated between $642-647 \mathrm{~nm}$ from $2 \mu \mathrm{M}$ red emitting QDs dissolved in toluene over a duration of 10 minutes under $20 \mathrm{~Hz}$ pumping at an intensity of $1.4 \mathrm{X}$ threshold $\left(30 \mu \mathrm{J} / \mathrm{mm}^{2}\right.$ per pulse). Error bars are obtained with 3 measurements. (F) Lasing spectra recorded from red emitting QDs dissolved in toluene at $\mathrm{t}=0 \mathrm{~min}$ and $\mathrm{t}=10 \mathrm{~min}$ show negligible photobleaching.

to lase (Fig. 1(A)). On the other hand, when the liquid has a higher refractive index, the WGM exists mainly in the liquid, which also provides optical feedback for lasing (Fig. 1(B)). Furthermore, the WGM can interact with a single molecular layer of gain molecules at the solid/liquid interface (Fig. 1(C)). ${ }^{9}$ For QD lasing in the aqueous environment (water refractive index $=1.33$ at visible wavelengths), we utilize the OFRRs first and third property described in Fig. 1(A) and (C). We also demonstrate QD lasing in toluene (refractive index=1.496 nm at visible wavelengths) using the OFRRs second property described in Fig. 1(B).

Commercially available QD solutions in aqueous buffer (Invitrogen Qdot ${ }^{\circledR} 655$; CdSe/ZnS quantum dots; 2 $\mu \mathrm{M}$ in borate buffer; $8 \times 15 \mathrm{~nm}$ average QD dimensions ${ }^{21}$ (Fig. 1(E))) and in toluene (Sigma; CdSeS/ZnS alloyed quantum dots; $2 \mu \mathrm{M}$ in toluene; $6 \mathrm{~nm}$ average $\mathrm{QD}$ diameter) were used in the experiments. Amine-to-amine cross-linking was used for surface immobilization of the Qdot ${ }^{\circledR} 655$ QDs on the inner surface of the OFRRs. For optofluidic QD FRET laser experiments, the covalent immobilization method was used to link Cy5 dye molecules to the surface of Qdot ${ }^{\circledR} 655$ QDs. Optical experiments were performed using a confocal setup exciting OFRRs with the output of a pulsed optical parametric oscillator (OPO) (repetition rate: $20 \mathrm{~Hz}$, pulse width: $5 \mathrm{~ns}$ ) (Fig. 1(D)). Excitation wavelength of $433 \mathrm{~nm}$ was used in all the QD experiments. A $25 \mathrm{~mm}$ focal distance planoconvex lens was used for focusing the excitation beam at a $0.8 \mathrm{~mm}$ spot on the OFRRs and collecting the OFRR laser emission. A spectrometer (Horiba 550) and a CCD camera were used for spectral detection of the OFRR laser emission signals. All measurements reported in this paper were performed in the absence of liquid flow, when the gain medium was kept still inside the OFRR. 

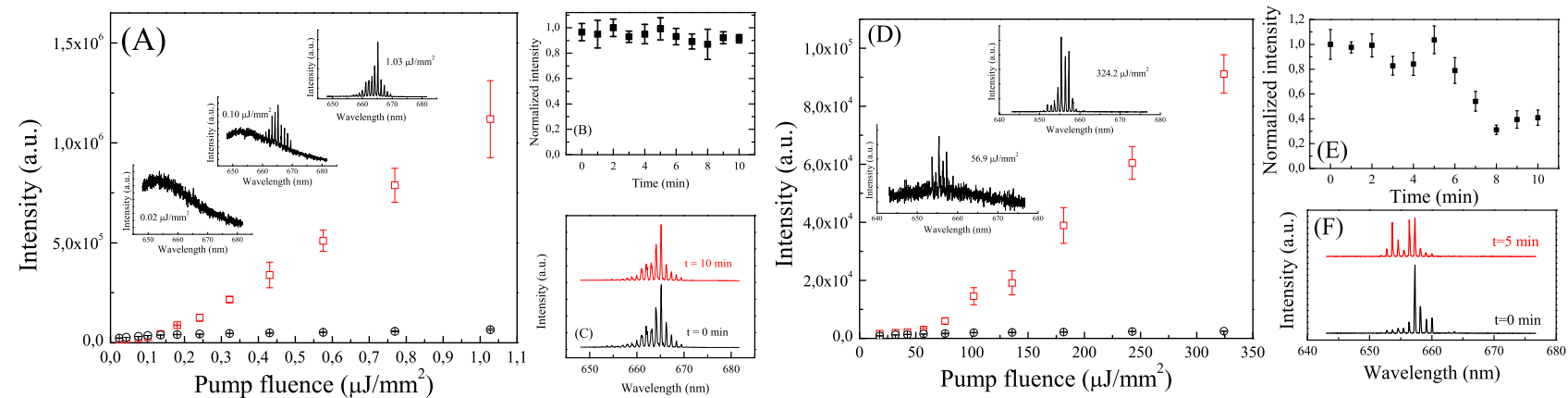

Figure 3. Spectrally integrated intensity as a function of pump intensity for laser emission (squares) and fluorescence (circles) for $21.6 \mu \mathrm{M}$ aqueous QDs in borate buffer solution. Spectral integration takes place in the range of 660-665 nm for lasing and 650-655 nm for fluorescence. The lasing threshold is $0.1 \mu \mathrm{J} / \mathrm{mm}^{2}$ per pulse. Insets show the examples of emission below and above the respective lasing threshold. Error bars are obtained with 3 measurements. (B) Normalized lasing intensity integrated between $660-670 \mathrm{~nm}$ from $2 \mu \mathrm{M}$ aqueous QDs over duration of 10 minutes under $20 \mathrm{~Hz}$ pumping at an intensity of around 10X threshold (1.06 $\mu \mathrm{J} / \mathrm{mm}^{2}$ per pulse). Error bars are obtained with 3 measurements. (C) QD lasing spectra recorded at $\mathrm{t}=0 \mathrm{~min}$ and $\mathrm{t}=10 \mathrm{~min}$ show negligible photobleaching. (D) Spectrally integrated intensity as a function of pump intensity for laser emission (squares) and fluorescence (circles) from a single layer of QDs immobilized on the inner surface of the ring resonator filled with PBS buffer solution. Spectral integration takes place in the range of 653-658 nm for lasing and 644-649 nm for fluorescence, respectively. The lasing threshold is $60 \mu \mathrm{J} / \mathrm{mm}^{2}$ per pulse. Error bars are obtained with 3 measurements. Insets show the examples of laser emission just and well above the lasing threshold. (E) Normalized lasing intensity integrated between 650-660 nm over duration of 10 minutes under $20 \mathrm{~Hz}$ pumping at an intensity of $3.5 \mathrm{X}$ threshold $\left(199 \mu \mathrm{J} / \mathrm{mm}^{2}\right.$ per pulse) from a single layer of aqueous QDs immobilized on the inner surface of an OFRR. Error bars are obtained with 3 measurements. (F) QD lasing spectra recorded at $t=0$ min and $\mathrm{t}=5 \mathrm{~min}$.

\section{LASING WITH QUANTUM DOTS DISSOLVED IN TOLUENE}

Fig. 2 summarizes the results of the lasing experiments we have performed with QDs dissolved in toluene, kept in OFRRs. Threshold pump intensities of 15 and $22 \mu \mathrm{J} / \mathrm{mm}^{2}$ were observed for orange (Fig. 2(A)) and red (Fig. 2(B)) emitting QDs dissolved in toluene, respectively. The observed lasing threshold pump intensities were much smaller than those reported in the other comparable study in the literature $\left(\sim 250 \mu \mathrm{J} / \mathrm{mm}^{2}\right)$ due to the superior properties of the OFRR as an optical cavity. ${ }^{22}$ In both cases, negligible photobleaching was observed during QD lasing for duations over 10 min using optical pumping intensities 1.4-3.5 higher than the corresponding threshold pump intensities (Fig. 2(C) and (D)).

\section{LASING WITH AQUEOUS QUANTUM DOTS}

Fig. 3(A) shows the QD emission spectra recorded at various pump intensities together with spectrally integrated intensities. At low pump intensities, only spontaneous emission is observed. With the increased pump intensity, multiple lasing peaks emerge at the red side of the QD emission band. An analysis of the power dependent spectral intensity for the lasing region between $660 \mathrm{~nm}$ and $665 \mathrm{~nm}$ plotted in Fig. 2 reveals a lasing threshold of $0.1 \mu \mathrm{J} / \mathrm{mm}^{2}$. In contrast, the non-lasing part $(650-655 \mathrm{~nm})$ of the QD emission spectrum increases sublinearly and saturation is observed at increased pump intensities. The observed lasing threshold is over 3 orders of magnitude lower than for the previously reported optofluidic laser (440-530 $\left.\mu \mathrm{J} / \mathrm{mm}^{2}\right)$ using aqueous microdroplet resonators that contained similar QD concentrations $(1.3-2.6 \mu \mathrm{M}) .{ }^{23}$ Such significant improvement is due to the excellent Q-factor of the OFRR $\left(\sim 10^{7}\right){ }^{18}$ The observed lasing threshold is also much lower than lasing threshold values reported in OFRR laser demonstrations using organic dyes and green fluorescent proteins of the similar concentration $(2-10 \mu \mathrm{M})\left(20-100 \mu \mathrm{J} / \mathrm{mm}^{2}\right) .{ }^{19}$ The high absorption cross section at the pump wavelength of the QDs contribute to the significant reduction in the lasing threshold in the QD laser over the dye or fluorescent protein based laser. Figs. 3(B) and (C) show the photostability of lasing intensity obtained from aqueous QDs at $2 \mu \mathrm{M}$ concentration during $10 \mathrm{~min}$, under a constant pump fluence of $0.7 \mu \mathrm{J} / \mathrm{mm}^{2}(7 \mathrm{X}$ the lasing threshold). Spectra recorded during this time show fluctuations of up to $30 \%$ in QD lasing intensity, but no clear sign of photobleaching. 


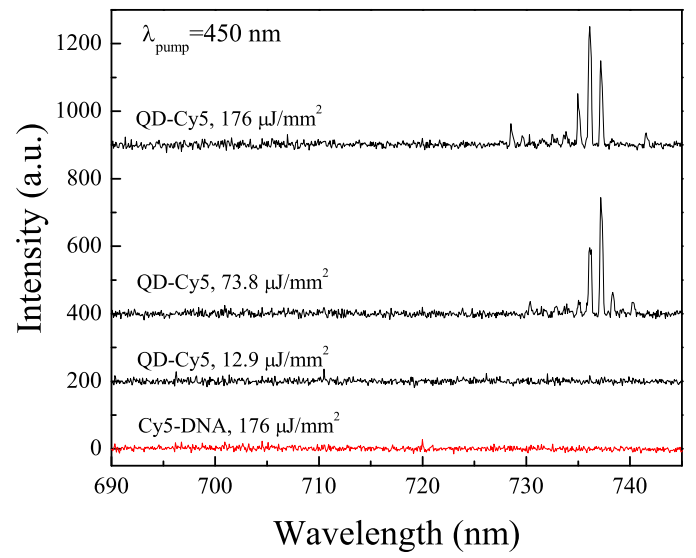

Figure 4. Emission spectra of QD-Cy5 and Cy5 when pumped at $450 \mathrm{~nm}$. Spectra are vertically shifted for clarity.
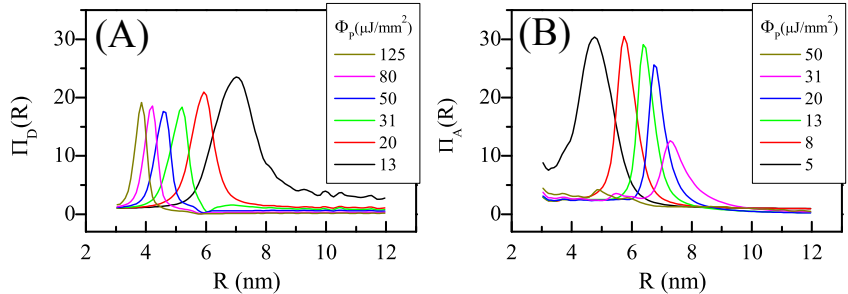

Figure 5. The sensitivity enhancement factor for (a) donor and (b) acceptor as a function of linker length $(R)$ at different $\Phi_{P}$ values. In all simulations, excitation pulse width was set to $\Delta \mathrm{t}=5 \mathrm{~ns}$ and the cavity Q-factor was $10^{6}$. Förster radius was assumed to be $6.1 \mathrm{~nm}$.

We performed surface immobilization biochemistry and attached a single layer of aqueous QDs on the inner surface of the OFRRs. OFRRs were subsequently filled with DI water. Upon optical pumping, photostable QD lasing was also observed for this case as demonstrated in Fig. 3(D)-(F). As compared to the results presented in Fig. 3(A)-(C) 2 and 3 using aqueous QD solutions, in this case lasing threshold fluence is larger $\left(\sim 50 \mu \mathrm{J} / \mathrm{mm}^{2}\right)$ and the average lasing wavelength $(\sim 655 \mathrm{~nm})$ is smaller. Both of these observations stem from the much smaller number of QDs coupled to the $\mathrm{WGMs}^{24}[48,49]$. However, despite the high pumping intensity conditions, a good photostability was still observed over the duration of $10 \mathrm{~min}$ for the case of lasing with a single QD layer (Fig. 3(E) and (F)).

Fig.4 shows the main results of the optofluidic QD FRET laser experiments. In these experiments an aqueous solution containing Qdot ${ }^{\circledR} 655$ QD-Cy5 dye molecules FRET pairs is used as the gain medium. The gain medium is pumped at $450 \mathrm{~nm}$ where considerable QD absorption exists while Cy5 absorption is very low. QD-Cy5 pairs revealed Cy5 lasing at a threshold pump intensity of around $12 \mu \mathrm{J} / \mathrm{mm}^{2}$ when the concentration of Cy5 molecules was measured to be $30 \mu \mathrm{M}$ from absorpbion measurements. In contrast, no lasing was observed from an aqueous solution of $30 \mu \mathrm{M}$ Cy5 molecules for pump powers up to $176 \mu \mathrm{J} / \mathrm{mm}^{2}$. These show that for the case of QD-Cy5 pairs, FRET served as the mechanism pumping the Cy5 acceptor molecules.

\section{PROSPECTS OF OPTOFLUIDIC LASERS FOR BIOCHEMICAL SENSING}

We carried out a comprehensive analysis of optofluidic FRET lasers using the framework of rate equations for the excited state populations of the donor and acceptor molecules and the corresponding donor and acceptor photon densities. Our rate equations are based on those developed previously for a dye laser consisting of a single-dye gain medium. ${ }^{25}$ These equations have been expanded to account for the presence of a saturable absorber dye acting as an energy acceptor which is radiatively coupled to the donor dye. ${ }^{26,27}$ Moreover, additional terms describing FRET-based non-radiative energy transfer between the donor and acceptor molecules have been included. ${ }^{28}$ 
Fig. 5 shows some key results of our analysis. In this figure the donor and acceptor sensitivity enhancement factors $\Pi_{D}$ and $\Pi_{A}$ are plotted as a function of the donor-acceptor linker length $(R) . \Pi_{D}$ and $\Pi_{A}$ quantify the enhancements observed in donor and acceptor emission intensities to $R$ for the case of an optofluidic laser as compared to the case of regular fluorescence. We define the sensitivity enhancement factor $\Pi$ as the ratio of lasing FRET sensitivity to the regular fluorescence FRET sensitivity, $\Pi=\Omega_{\text {lasing }}(R) / \Omega_{\text {fluorescence }}(R)$. Here, $\Omega_{\text {lasing }}(R)=\left|\left(100 \times d E_{\text {out }}\right) /\left(E_{\text {out }} d R\right)\right|$ is the sensitivity of the output energy of stimulated emission from the donor or acceptor placed inside a high-Q cavity to changes in $R$ characterizing the gain medium whereas $\Omega_{\text {fluorescence }}(R)$ is the corresponding sensitivity of the output energy of regular (non-amplified) fluorescence from the donor or acceptor placed inside a cavity with $Q=1$. $E_{\text {Dout }}$ and $E_{\text {Aout }}$ represent the donor or acceptor emission energies. As illustrated by Fig. 5(a) and (b), maximal enhancement factors of $\sim 20$ for donor and $\sim 25$ for acceptor sensitivities are observed for a relatively broad range of $R$ at different pump fluences $\Phi_{P}$. The results of our calculations are in good agreement with the experiments reported in Ref. ${ }^{7}$ where optofluidic lasers were shown to be 16 times more sensitive to $\mathrm{Mg}^{2+}$ concentration in DNA Halliday junctions.

\section{CONCLUSION}

By combining the excellent fluorescent properties of the state-of-the-art core/shell QDs with the unique properties of the OFRR as an optical cavity, we demonstrated optofluidic QD lasing using aquesous solutions and toluene as the solvent. We also demonstrated capability of QDs as donors in FRET lasers. These results greatly improve the versatility for optofluidic laser operation due to the broad and large absorption cross-section of QDs in the blue and UV range. Free from limitations posed by photobleaching, such optofluidic QD lasers are directly applicable to numerous applications in bioanalysis, where optofluidic dye lasers have already proven their superiority over spontaneous fluorescent emission-based measurements. Finally, we have presented a comprehensive theoretical analysis of optofluidic lasers with gain media formed by a FRET pair of donor and acceptor dyes and showed that FRET lasing can lead to more than 20-fold enhancement in detection sensitivities of conformation changes for linker lengths in the Förster radius range.

\section{ACKNOWLEDGMENTS}

The authors are grateful for the support from TÜBİTAK. A.K. acknowledges the support from a Fulbright Fellowship and the University of Michigan as a visiting scholar. Q.C. and X.F. acknowledge the support from the National Institutes of Health (1R21EB016783). The authors thank M. Tanyeri, M.-A. Mycek, S. Sivaramakrishnan, and E. Özelci for their help.

\section{REFERENCES}

[1] Fan, X. and Yun, S. H., "The potential of optofluidic biolasers," Nat. Methods 11(2), 141-147 (2014).

[2] Fan, X. and White, I. M., "Optofluidic microsystems for chemical and biological analysis," Nat. Photonics 5(10), 591-597 (2011).

[3] Sun, Y. and Fan, X., "Distinguishing DNA by analog-to-digital-like conversion by using optofluidic lasers," Angew. Chem. Int. Ed. 51(5), 1236-1239 (2012).

[4] Chen, Q., Zhang, X., Sun, Y., Ritt, M., Sivaramakrishnan, S., and Fan, X., "Highly sensitive fluorescent protein FRET detection using optofluidic lasers," Lab Chip 13(14), 2679-2681 (2013).

[5] Jonáš, A., Aas, M., Karadag, Y., Manioglu, S., Anand, S., McGloin, D., Bayraktar, H., and Kiraz, A., "In vitro and in vivo biolasing of fluorescent proteins suspended in liquid microdroplet cavities," Lab Chip 14, 3093-3100 (2014).

[6] Polson, R. C. and Vardeny, Z. V., "Random lasing in human tissues," Appl. Phys. Lett. 85, 1289-1291 (2004).

[7] Zhang, X., Lee, W., and Fan, X., "Bio-switchable optofluidic lasers based on DNA holliday junctions," Lab Chip 12(19), 3673-3675 (2012).

[8] Wu, X., Khaing Oo, M. K., Reddy, K., Chen, Q., Sun, Y., and Fan, X., "Optofluidic laser for dual-mode sensitive biomolecular detection with a large dynamic range," Nat. Commun. 5, 3779 (2014). 
[9] Chen, Q., Ritt, M., Sivaramakrishnan, S., Sun, X., and Fan, X., "Optofluidic lasers with a single molecular layer of gain," Lab Chip 14(24), 4590-4595 (2014).

[10] Pellegrino, T., Manna, L., Kudera, S., Liedl, T., Koktysh, D., Rogach, A. L., Keller, S., Ra1dler, J., Natile, G., and Parak, W. J., "Hydrophobic nanocrystals coated with an amphiphilic polymer shell: A general route to water soluble nanocrystals," Nano Lett. 4, 703-707 (2004).

[11] Leatherdale, C. A., Woo, W.-K., Mikulec, F. V., and Bawendi, M. G., "On the absorption cross section of cdse nanocrystal quantum dots," J. Phys. Chem. 106, 76197622 (2002).

[12] Michalet, X., Pinaud, F. F., Bentolila, L. A., Tsay, J. M., Doose, S., Li, J. J., Sundaresan, G., Wu, A. M., Gambhir, S. S., and Weiss, S., "Quantum dots for live cells, in vivo imaging, and diagnostics," Science 307(5709), 538-544 (2005).

[13] Resch-Genger, U., Grabolle, M., Cavaliere-Jaricot, S., Nitschke, R., and Nann, T., "Quantum dots versus organic dyes as fluorescent labels," Nat. Methods 5(9), 763-775 (2008).

[14] Wu, C.-S., Khaing Oo, M. K., and Fan, X., "Highly sensitive multiplexed heavy metal detection using quantum-dot-labeled dnazymes," ACS Nano 4, 5897-5904 (2010).

[15] Kiraz, A., Chen, Q., and Fan, X., "Optofluidic lasers with aqueous quantum dots," ACS Photonics 2(6), 707-713 (2015).

[16] Chen, Q., Kiraz, A., and Fan, X., "Optofluidic fret lasers using aqueous quantum dots as donors," Lab Chip 16, 353-359 (2016).

[17] Aas, M., Chen, Q., Jonas, A., Kiraz, A., and Fan, X., "Optofluidic fret lasers and their applications in novel photonic devices and biochemical sensing," Selected Topics in Quantum Electronics, IEEE Journal of 22(4), 1-15 (2016).

[18] Shopova, S. I., Cupps, J. M., Zhang, P., Henderson, E. P., Lacey, S., and Fan, X., "Opto-fluidic ring resonator lasers based on highly efficient resonant energy transfer," Opt. Express 15(20), 12735-12742 (2007).

[19] Lacey, S., White, I. M., Sun, Y., Shopova, S. I., Cupps, J. M., Zhang, P., and Fan, X., "Versatile opto-fluidic ring resonator lasers with ultra-low threshold," Opt. Express 15, 17433-17442 (2007).

[20] White, I. M., Gohring, J., Sun, Y., Yang, G., Lacey, S., and Fan, X., "Versatile waveguide-coupled optofluidic devices based on liquid core optical ring resonators," Appl. Phys. Lett. 91, 241104 (2007).

[21] Giepmans, B. N. G., Deerinck, T. J., Smarr, B. L., Jones, Y. Z., and Ellisman, M. H., "Correlated light and electron microscopic imaging of multiple endogenous proteins using quantum dots," Nat. Methods 2(10), 743-749 (2005).

[22] Wang, Y., Leck, K. S., Ta, V. D., Chen, R., Nalla, V., Gao, Y., He, T., Demir, H. V., and Sun, H., "Blue liquid lasers from solution of cdzns/zns ternary alloy quantum dots with quasi-continuous pumping," Advanced Materials 27(1), 169-175 (2015).

[23] Schäfer, J., Mondia, J. P., Sharma, R., Lu, Z. H., Susha, A. S., Rogach, A. L., and Wang, L. J., "Quantum dot microdrop laser," Nano Lett. 8, 1709-1712 (2008).

[24] Aas, M., Jonáš, A., and Kiraz, A., "Lasing in optically manipulated, dye-doped emulsion microdroplets," Opt. Commun. 290, 183-187 (2013).

[25] Atkinson, J. and Pace, F., "The spectral linewidth of a flashlamp-pumped dye laser," IEEE J. Quantum Electron. 9(6), 569-574 (1973).

[26] Hebling, J., "20 ps pulse generation by an excimer laser pumped double self-Q-switched distributed feedback dye laser," Appl Phys B 47(3), 267-272 (1988).

[27] Hebling, J., Seres, J., Bor, Z., and Racz, B., "Dye laser pulse shortening and stabilization by Q-switching," Opt Quant Electron 22(4), 375-384 (1990).

[28] Ahamed, B. and Palanisamy, P., "Nd:YAG laser pumped energy transfer distributed feedback dye laser in Rhodamine 6G and Acid blue 7 dye mixture," Optics Communications 213(1-3), 67-80 (2002). 